\title{
@@ OPENACCESS FROZEN SHOULDER: OUTCOME OF INTRA-ARTICULAR \\ Check for updates STEROID INJECTION IN IDIOPATHIC ADHESIVE CAPSULITIS IN TERMS OF PAIN RELIEF AND RANGE OF MOTION
}

Department of Orthopedic \& Spine Unit, Hayatabad Medical Complex (Medical Teaching Institute), Peshawar-Pakistan

Address for correspondence: Muhammad Ibrahim Department of Orthopedic \& Spine Unit, Hayatabad Medical Complex (Medical Teaching Institute), Peshawar-Pakistan

E-mail:

ibrahim9996@gmail.com

Date Received:

January, $12^{\text {th }} 2021$

Date Revised:

October, $6^{\text {th }} 2021$

Date Accepted:

October, $6^{\text {th }} 2021$

Muhammad Waqar Khan, Muhammad Ibrahimª, Sanaullah, Imran Khan, Zeeshan Khan, Muhammad Arif Khan

\section{ABSTRACT}

Objective: To find out the outcomes of intra articular steroid injection in idiopathic adhesive capsulitis, in terms of pain control and functional activity.

Methodology: This Quasi experimental study was conducted in the Department of Orthopedic and Spine, Hayatabad Medical Complex, from February 2019 to February 2020. All patients aged above 18 years of age, both genders, having a diagnosis of idiopathic adhesive capsulitis were included in this study. A total of 200 patients were included in the study. Sample size was calculated by Open-Epi. Their pre injection Shoulder Pain and Disability Index (SPADI) and Visual Analogue Score (VAS) were recorded. All patients received intra-articular corticosteroid injection. SPADI and VAS were recorded after 4 and 12 weeks. The baseline scores and those recorded at follow up after injection were compared. Data was analyzed using SPSS version 23.

Results: Out of 200, 86 (43\%) were female and $114(57 \%)$ were male. The average age of our patients was 50.85 $( \pm 7.36)$. Their average pre injection SPADI was $79( \pm 6.4)$ while the average VAS was $6.5( \pm 0.9)$. Mean evaluation scores at 4th week of intra-articular corticosteroid injections were improved to SPADI $27.32( \pm 3.14)$ and VAS was $2.55( \pm 1.1)$ with a statistically significant $p$-value of 0.0001 . At 12 th week SPADI disability score was still consistent at $27( \pm 3)$ but their VAS score worsened to $6.1( \pm 0.86)$.

Conclusion: Patients suffering from idiopathic adhesive capsulitis can benefit from intra-articular corticosteroid injection alone. The range of motion and functional ability in their diseased shoulder can get better till 12 weeks. Their pain, on other hand, will get better initially but later on it'll re-appear and would warrant re-evaluation and change in management plan.

Keywords: Adhesive capsulitis; Intraarticular injections; Visual analog scale; Disability Index.

\section{INTRODUCTION}

Frozen shoulder, also known as Adhesive capsulitis, is a commonly known cause of pain and stiffness in shoulder especially in those over 50 years of age and is therefore also known as " 50 shoulder" in China. Prevalence in western countries has been reported to be $2-5 \%$ in general population and its more common among women. ${ }^{2}$ The disease is more common among patients having hyperthyroidism, hyperlipidemia or diabetes. Diabetic patients are almost $11-30 \%$ more prone to develop adhesive capsulitis than general population. $^{3}$

It is characterized by substantial decrease in active and passive mobility of the shoulder joint along with pain. The most crucial loss is in the external rotation and abduction of the joint. ${ }^{4}$ The pathology underneath leads to reduction in joint cavity by thickening of inferior capsule ${ }^{5}$ and contracture of the rotator interval, coraco-humeral ligament and anterior capsule with a combination of synovial inflammation and capsular fibrosis. ${ }^{6}$ The disease progression has been divided into three clinical phases; the initial phase where pain is the major symptom lasting from 3 to 9 months, followed by a freezing phase with progressive stiffness lasting from 4 to 12 months and finally, the recovery phase with gradual return of movement, lasting 5-26 months. ${ }^{7}$ Although adhesive capsulitis is thought to be self-limiting, many patients do not achieve full recovery. Favejee et al report that after 4 years $59 \%$ of patients attain full recovery. ${ }^{8}$ While Reeves, in a prospective study of 41 patients with 5 to 10 years' follow-up, found that only $39 \%$ of patients had full recovery without having any treatment. ${ }^{9}$ 
There are a number of treatment options being offered to patients these days. These include non-steroidal anti-inflammatory drugs (NSAIDs) or other analgesics, physical therapy, manipulation under anesthesia (MUA), dilation or distension of the capsule, arthroscopic or open capsular release, and injections of sodium hyaluronate or corticosteroids. ${ }^{10}$ Although there is no definitive treatment ${ }^{2}$, management strategy depends upon the severity of the symptoms and whether or not the patient is suffering from idiopathic adhesive capsulitis or secondary adhesive capsulitis. In secondary adhesive capsulitis it is recommended to address the primary pathology first to achieve satisfactory results. ${ }^{11}$

One study by Buchbinder et al reports from their systematic review of randomized and pseudo-randomized control trials of injection of corticosteroid for shoulder pain that corticosteroid injection might be helpful. ${ }^{12}$ Griesser et al did a systematic review of randomized-controlled trials(RCTs), they came to the conclusion that intra-articular corticosteroid injections leads to greater improvement in pain relief and ROM not only in short term but also in long term, but compared to other treatments the results were similar in the long term. ${ }^{13}$ Another systematic review reports that intrarticular steroids may prove beneficial in the terms of reducing pain and disability in the early 6 weeks but beyond 6 weeks, they reported, no significant benefit as compared to physical therapy alone. ${ }^{10}$ While a meta-analysis by Wei wang et al reports that although ROM improves both in short term and long term follow-up but VAS pain score improves initially but in long term follow-up they reported no significant difference comparing with control. ${ }^{14}$

The existing practice of administering the steroid injection(s) is a valid and tested option in management of Idiopathic steroid injection. The current study tests the effects of a single dose of intra-articular steroid injection, in terms of pain control and functional outcome at short-term and long-term follow-up. We wanted to bridge the gap between the existing practice and the actual effects of the injection.

\section{METHODOLOGY}

After approval from ethical review board, this quasi experimental study conducted in the Department of Orthopedic and Spine, Hayatabad Medical Complex, Peshawar from February 2019 to February 2020 with sample size of 200, calculated through Open-epi. These patients were selected by non-probability consecutive sampling technique. All patients above 18 years of age, having a clinical diagnosis of idiopathic adhesive capsulitis were included in this study. Patients having symptoms in one joint for atleast 3 months, but less than 2 years were included in this study. ${ }^{15}$ Patients included in this study had limited passive range of motion (PROM) with a limitation of more than $30 \%$ of two (out of three) shoulder movements and none of the three movements (Abduction $=A B D$, External rotation $=\mathrm{ER}$ and Internal rotation $=\mathrm{IR}$ ) were normal. ${ }^{3}$ Patients who were diagnosed to have a secondary adhesive capsulitis or had history of intraarticular injection on the same joint previously were excluded from the study.

For data collection, Shoulder pain and disability index (SPADI) and Visual analogue scale was used. The Shoulder Pain and Disability Index (SPADI) is a 13-item patient-completed questionnaire that assesses pain level and difficulties with activities of daily living (ADLs) that require the use of the upper extremities. The Disability subscale contains eight items, whereas the pain subscale has five. Total score of SPADI is 100. It is a most reliable tool with ICC value of 0.90 . Second outcome measure used was visual analogue scale (VAS). A Visual Analogue Scale (VAS) is a measurement tool that attempts to quantify a feature or attitude that is thought to range throughout a continuum of values but is difficult to measure directly. It's frequently used in epidemiologic and clinical studies to assess the severity or frequency of certain symptoms. The degree of discomfort a patient experiences, for example, might range from none to a great deal. It is a reliable tool for measuring pain with test retest reliability of 0.94 .

All the patients of adhesive capsulitis were explained the purpose and benefit of this study and written informed consent was taken. All patients were subjected to detailed history, examination and appropriate base line investigations including a simple shoulder AP view $\mathrm{x}$-ray to exclude any secondary causes. A questionnaire was filled out for every patient by the researcher himself which inquired about their primary symptom and duration of symptoms.

Their pre injection Shoulder Pain and Disability Index (SPADI) and Visual Analogue Score (VAS) score was recorded. All patients received intra-articular corticosteroid injection. This injection was made by the researcher himself by mixing $2 \mathrm{ml}(80 \mathrm{mg})$ of methylprednisolone and $1 \mathrm{ml}$ lignocaine $2 \%$ (for local pain relief due to intra-articular injection). ${ }^{16}$ This injection was administered in a single syringe under aseptic protocol. Patients were sent home after completion of the procedure and advised range of movement exercises. Patients were asked for follow up at 4 weeks (short-term) and at 8-12 weeks (long term follow-up). At both of the follow-ups their SPADI and VAS scores were re-evaluated.

All the data was analyzed with SPSS v.23. Mean and standard deviation was computed for continuous variables like Age and Gender. Frequency and percentage was computed for categorical variables like VAS scoring for pain and SPADI scoring for range of motion and functionality. ${ }^{3}$ Within group analysis was done through parametric paired sample 
t-test through which $p$-value was calculated.

\section{RESULTS}

After sorting our patients through inclusion and exclusion criteria, the total number of patients registered for this study were 200. Out of which $86(43 \%)$ were female and $114(57 \%)$ were male. The mean age of patients was $50.85( \pm 7.36)$. The patients in current study were diagnosed to have adhesive capsulitis predominantly in the right shoulder $58 \%$, while $42 \%$ presented with left shoulder pathology. Using SPADI baseline disability score, patients were evaluated and their average score was calculated to be $79( \pm 6.4)$ while the average baseline VAS score of these patients was $6.5( \pm 0.9)$. Results of both variables were statisitically significant at $4^{\text {th }}$ week evaluation as their $p$-value was 0.001 ( $p$-value $<0.05$ ). Mean evaluation scores at $4^{\text {th }}$ week of intra-articular corticosteroid injections were improved to SPADI $27.32( \pm 3.14)$ and VAS was 2.55 $( \pm 1.1)$ with a statistically significant $p$-value of 0.001 . At $12^{\text {th }}$ week these patients were re-evaluated and their mean SPADI and VAS scores were recorded, results of SPADI were highly statistically significant as their $p$-value was 0.001 ( $p$-value $<0.05$ ), but results of VAS was statistically insignificant as their $p$-value was 0.164 ( $p$-value>0.05). Their mean SPADI disability score was still consistent at $27( \pm 3)$ with $p$-value of 0.001 but their VAS score worsened to $6.1( \pm 0.86)$ with P-value of 0.164 as show in Table 1.

\section{DISCUSSION}

Adhesive capsulitis is a very common problem presenting to an orthopedic sur- geon's clinic. These patients usually are in mild to moderate pain with stiffness which hampers their ability to perform their daily life activities. Usually adhesive capsulitis is known to be a self-limiting disease with full recovery of ROM and pain. However, there are many patients who live with this disease for years. In one study they reported that $50 \%$ of their patients had symptoms of pain and stiffness in their shoulder for an average of 7 years. ${ }^{17}$ This long-term pain and stiffness limits a patient's daily life and work activities making them dependent on others in severe cases.

This disease has been reported to be more common in women ${ }^{18}$ our results indicate more prevalence among males, which is comparable to a study previously done in the same unit. ${ }^{16}$ Previous studies indicate that the left shoulder joint is more commonly involved than right $^{18}$ but our patients presented more commonly with the pathology in their right shoulder.

The severity of this disease can be judged by our initial SPADI disability index and VAS scores $79( \pm 6.4)$ and $6.5( \pm 0.9)$, respectively. These baseline scores are comparable to scores reported by Buchbinder et al. baseline SPADI score 64.7 (14.1) and VAS score 7.4 (1.4). ${ }^{19}$ Similarly, Siraj M et al. reported their baseline SPADI disability index scores to be $79.5( \pm 7.6)^{16}$, showing the severity of the disease at presentation. This indicates that these patients are in much pain at initial presentation and their problems can only to get worse if they are not properly diagnosed and managed. This disease can usually be treated with conservative management and a person can reach up to full range of motion with rehabilitation, thus surgery is seldom needed. The option of treating a patient of adhesive capsulitis with intra-articular corticosteroid injection has already proven benefits in short term follow-up but we wanted to see the effects of it in long term follow up.

After initial intervention we have seen marked improvement in our patients pertaining to their range of motion and pain at short term follow up (4 weeks), their scores remain the same even at their second long term follow-up (12 weeks) TABLE. Although their VAS scores improved initially significantly, the intra-articular steroid injection could not sustain the pain relief at the last long-term follow-up TABLE. These results are comparable with studies published before. ${ }^{3,14}$ Song. A et al. also reported in their systematic review a 60\% decrease in VAS scores and improvement in ROM at early follow-up but at long-term follow-up their results were similar to other treatment options including placebo. ${ }^{10}$ Clinical significance: We would like to show our readers, using these results, that even a single steroid injection can benefit patient. They'll have good functional activity even at long-term follow-up but their pain control would've have re-appeared by then and they'll need to be prescribed accordingly for the pain management.

The limitations in our study were that there was no control group and the patients were not randomized. Secondly, the intra-articular steroid injection was inserted without ultrasound guidance and that increases the chance of human error. We hope that future studies can conduct a double-blinded RCT to limit bias as much as possible and include a radiologist's help to administer an ultrasound

Table 1: Short-term and Long-term results of intra-articular steroid injection in idiopathic adhesive capsulitis.

\begin{tabular}{|c|c|c|c|c|c|}
\hline & At presentation & $\begin{array}{l}\text { At short-term fol- } \\
\text { low-up (4th week) }\end{array}$ & P-Value & $\begin{array}{l}\text { At long-term fol- } \\
\text { low-up (12th week) }\end{array}$ & P-Value \\
\hline Shoulder Pain and Disability Index (SPADI) & $79( \pm 6.4)$ & $27.32( \pm 3.14)$ & $0.001^{*}$ & $27( \pm 3)$ & $0.001^{*}$ \\
\hline
\end{tabular}

*=Statistically Significant Value 
guided intra-articular steroid injection to limit the chances of human error.

\section{CONCLUSION}

Our research concludes that the patients suffering from idiopathic adhesive capsulitis can benefit from intra-articular corticosteroid injection alone. Their range of motion can get better and allows the patient to regain functional ability in their diseased shoulder till 12 weeks. Their pain, on other hand, will get better initially but later on it'll re-appear and would warrant re-evaluation and change in management plan.

\section{REFERENCES}

1. Shang X, Zhang Z, Pan X, Li J, Li Q. Intra-articular versus subacromial corticosteroid injection for the treatment of adhesive capsulitis: A meta-analysis and systematic review. BioMed Res Int. 2019; 15: 2019.

2. Cho $\mathrm{CH}$, Bae KC, Kim DH. Treatment Strategy for Frozen Shoulder. Clin Orthop Surg. 2019;11(3):249-57.

3. Sharma SP, Bærheim A, Moe-Nilssen $R$, Kvåle A. Adhesive capsulitis of the shoulder, treatment with corticosteroid, corticosteroid with distension or treatment-as-usual; a randomised controlled trial in primary care. BMC Musculoskelet Disord. 2016;17:232.
4. Shah N, Lewis M. Shoulder adhesive capsulitis: systematic review of randomised trials using multiple corticosteroid injections. $\mathrm{Br} J$ Gen Pract. 2007;57(541):662-7.

5. Wiley AM. Arthroscopic appearance of frozen shoulder. J Arth Rel Sur. 1991;7(2):138-43.

6. Hsu JE, Anakwenze OA, Warrender WJ, Abboud JA. Current review of adhesive capsulitis. J Sho Elb Sur. 2011;20(3):502-14.

7. Hannafin JA, Chiaia TA. Adhesive capsulitis. A treatment approach. Clin Orth Rel Res. 2000; (372):95-109.

8. Favejee M, Huisstede B, Koes B. Frozen shoulder: the effectiveness of conservative and surgical interventionssystematic review. $\mathrm{Br} J$ Spo Med. 2011;45(1):49-56.

9. Reeves B. The natural history of the frozen shoulder syndrome. Scan J Rheu. 1975; 4(4):193-6.

10. Song A, Higgins LD, Newman J, Jain NB. Glenohumeral corticosteroid injections in adhesive capsulitis: a systematic search and review. PM \& R. 2014;6(12):1143-56.

11. D'Orsi GM, Via AG, Frizziero A, Oliva F. Treatment of adhesive capsulitis: a review. Mus Lig Tend J 2012;2(2):70-8.

12. Buchbinder R, Green S, Youd JM. Corticosteroid injections for shoulder pain. Coch Data Sys Rev. 2003(1).
13. Griesser MJ, Harris JD, Campbell JE, Jones GL. Adhesive capsulitis of the shoulder: a systematic review of the effectiveness of intra-articular corticosteroid injections. JBJS. 2011; 93(18):1727-33.

14. Wang W, Shi M, Zhou C, Shi Z, Cai X, Lin $T$, et al. Effectiveness of corticosteroid injections in adhesive capsulitis of shoulder: A meta-analysis. Med. 2017;96(28):7529.

15. Tveitå EK, Tariq R, Sesseng S, Juel NG, Bautz-Holter E. Hydrodilatation, corticosteroids and adhesive capsulitis: A randomized controlled trial. BMC Mus Disord. 2008;9(1):53.

16. Siraj M, Anwar W, Iqbal MJ. Effectiveness of intra-articular corticosteroid injection in the treatment of idiopathic frozen shoulder. J Surg Pak. 2012;17:57-60.

17. Shaffer B, Tibone JE, Kerlan RK. Frozen shoulder. A long-term follow-up. J Bon Joint Surg Am. 1992;74(5):738-46.

18. Tasto JP and Elias DW. Adhesive capsulitis. Spo Med Arth Rev. 2007;15(4):21621.

19. Buchbinder R, Hoving JL, Green S, Hall S, Forbes A, Nash P. Short course prednisolone for adhesive capsulitis (frozen shoulder or stiff painful shoulder): a randomised, double blind, placebo controlled trial. Ann Rheum Dis. 2004;63(11):1460-9.

\section{Author's Contribution}

WK designed the study, did the data acquisition, drafted the manuscript and critically reviewed the manuscript. MI, SU, IK and ZK helped in data acquisition, drafting and critically reviewed the manuscript. MAK supervised the study and critically reviewed the manuscript. Authors agree to be accountable for all aspects of the work in ensuring that questions related to the accuracy or integrity of any part of the work are appropriately investigated and resolved. 